\title{
The development of a light and color teaching kit for astronomy
}

Robert Sparks, Constance Walker, Stephen Pompea

Robert T. Sparks, Constance E. Walker, Stephen M. Pompea, "The development of a light and color teaching kit for astronomy," Proc. SPIE 8481, Optics Education and Outreach II, 84810C (15 October 2012); doi: 10.1117/12.929930

SPIE Event: SPIE Optical Engineering + Applications, 2012, San Diego, California, United States 


\title{
The Development of a Light and Color Teaching Kit For Astronomy
}

\author{
Robert T. Sparks, Constance E. Walker, Stephen M. Pompea \\ National Optical Astronomy Observatory, 950 N. Cherry Ave, Tucson, AZ USA 85719
}

\begin{abstract}
Astronomy is an observational science. The data astronomers collect is primarily in the form of light emitted by astronomical objects across the electromagnetic spectrum. The Education and Public Outreach Group at the National Optical Astronomy Observatory has developed a low-cost kit for teaching about light and color in the context of astronomy.
\end{abstract}

We will present an outline of the activities and materials included in the kit as well as the pedagogical justification for the scope and sequence. We will present the initial feedback we have received on the activities as well as future plans for dissemination.

Keywords: Education, Astronomy, High School, Middle School, Instructional Materials

\section{INTRODUCTION}

Hands-On Optics (HOO) was a National Science Foundation (NSF) funded program designed to develop optics activities for middle school students ${ }^{1}$. HOO was a collaboration between the National Optical Astronomy Observatory, the International Society for Optical Engineers (SPIE) and the Optical Society of America (OSA). The HOO program developed a series of six optics modules, each containing activities covering a different areas of optics, to be used in informal science education venues such as after school programs and science centers. The modules were targeted at the middle school level but have been adapted for use with older and younger students as well. The activities were designed to be hands-on and appeal to non-science students.

The HOO materials have been adapted for use in a variety of different settings including Astronomy From the Ground Up (AFGU), a collaboration with the Astronomical Society of the Pacific, Laserfest, a celebration of the $50^{\text {th }}$ Anniversary of the invention of the laser, and the International Year of Astronomy with the Galileoscope. ${ }^{2}$ In each of these collaborations we developed a low cost kit that can be used by teachers, graduate students and outreach professionals in their educational outreach activities. Both of these kits have been used by SPIE with their student chapters and we have taught short courses at SPIE meetings on their use.

In the spring of 2011, NOAO set about developing a new kit that could be used in venues such as SPIE short courses and short courses for high school teachers. Building on a variety of activities, we decided to look at the topics of Light and Color in Astronomy. Light (of all wavelengths) is the primary tool astronomers have for gathering information on astronomical objects. Additionally, there are many commonly held misconceptions about the nature of light and color that we want to address through education. ${ }^{3}$ A thorough understanding of light and color is essential to understanding astronomy.

The kit was developed in a similar manner to the HOO kits. We use a variety of hands-on activities and interactive demonstrations to illustrate various properties of light and color The activities are designed to be used in informal settings and targeted at students in the fifth through eighth grade and use accepted best practices in hands-on optics education. ${ }^{4,5}$ The kits are targeted at students in fifth through eighth grade but many of the activities can be used with older or younger students with slight modifications. The demonstrations and activities are designed to be playful, exploratory and appeal to non-science students.

\section{THE LIGHT AND COLOR KIT CONTENTS}

Optics Education and Outreach II, edited by G. Groot Gregory, Proc. of SPIE Vol. 8481

84810C · (c) 2012 SPIE · CCC code: 0277-786/12/\$18 · doi: 10.1117/12.929930 
The kit was designed to be low cost so they could be distributed free or at low cost to teachers. We also elected not to supply some materials that are present in most classrooms (scissors, rulers, etc) to keep the cost down and the production of the kits relatively simple. The kit that we designed contains:

- 1 Avon Derma-Spec Skin Imager (ultraviolet light)

- 1 piece of 6"x6" diffraction grating film

- 15 pairs of diffraction grating glasses

- 1 red LED keychain light

- 1 blue LED keychain light

- 1 Write \& See Pad

- $\quad$ Bag of 250 UV Sensitive beads

- 1 Tube of glow bracelets

- 1 bag of Wint-O-Green Lifesavers

- 4 invisible ink pens

- 1 set of color filters

- 1 roll of cash register tape

- 1 Light And Color in the Night Sky CD

The cost of these materials is approximately $\$ 60$.

\section{THE ACTIVITIES}

The goal of this kit is to illustrate how light behaves and how astronomers use light to learn about the universe. Each of the activities includes connections to astronomy and questions to inspire student discussion about extracting information from light. The activities are outlined in this section.

\subsection{What is White Light}

In this activity, students explore the nature of white light. An overhead transparency projector is used to project white light onto the wall. A couple of folders are placed on projector to create a small slit and a diffraction grating is taped to the exit pupil of the projector (see image).

This setup produces a spectrum of light on the wall. Students discuss the source of the colors and observe the order of the colors. You can use the diffraction grating glasses to look at the white light source as well.

Students start exploring color with the color filters. The kit includes the three primary color filters (red, green and blue) and the three secondary color filters (cyan, yellow, magenta). Students predict how the spectrum will change when you add each color filter. The teacher discusses how you can combine any two of the primary colors to make a secondary color (i.e. red and green make yellow). When the secondary color filters are placed on the projector, students are frequently surprised to see the filter allows two primary colors to pass through the filter.

The teacher discusses how astronomers use color filters to take images and analyze astronomical objects.

\subsection{The Spectra of Lights}

The Spectra of Lights activity was adapted from NOAO's GLOBE at Night Dark



Figure 1: An overhead projector configured to project a spectra.

Sky Rangers Activity of the same name ${ }^{6}$. This activity lets students explore the spectra of different types of lights. The kit includes 15 pairs of diffraction grating glasses and the teacher supplies the lights to be analyzed. The lights can include incandescent lights, fluorescent lights (including compact fluorescent), LED's, colored lights, neon lights, black lights, etc.

Students will observe that different lights produce different spectra. Some are continuous and some are line spectra. A good addition is to observe lights with different temperatures to see the differences in the spectra. Using a variety of 
different colored light bulbs, compact fluorescent bulbs and LED lights show a wide variety of spectra with different features. Both emission line spectra and continuous spectra are observed with the glasses. Teachers discuss the different ways the spectra are produced and can add information about the energy efficiency of each type of bulb and make connections to eco-photonics ${ }^{7,8}$. Connections to dark skies are included since shorter wavelength light scatters more and interferes more with astronomical observations.

\subsection{Catch the Wave}

Catch the Wave is based on a Hands-On Optics activity and focuses on the wave nature of light. Students use a long spring to observe waves and learn several of the properties of waves including wavelength, frequency, and amplitude. Students will learn that the different colors of light have different wavelengths (and frequencies). The spring stretches across the room and students can easily produce waves with a variety of properties. Students can produce the fundamental wave and several harmonics illustrating the inverse relationship between frequency and wavelength.

\subsection{Scaling the Electromagnetic Spectrum}

This activity is adapted from our Hands-On Optics program. Students make a scaled model of different wavelengths of light ranging from UVC light to thermal infrared. Students see the wide range of wavelengths covered by this portion of the EM spectrum. The scale is one million times larger than actual size so the models they build range from $25 \mathrm{~cm}$ (for UVC light) to 10 meters (for thermal infrared). We chose the specific wavelengths used in our model to relate to types of waves students would be familiar with or produce interesting effects that can be discussed. We included UVC (which can kill bacteria), UVB (which causes sunburn), UVA (which can cause skin damage), blue light, red light, $1,000 \mathrm{~nm}$ (close to the wavelength used by infrared remote controls) and $10,000 \mathrm{~nm}$ (thermal infrared used by night vision goggles). This activity can be extended mathematically to show why radio waves, X-rays and gamma rays are not included. Radio waves would be far too long to fit in a classroom (a three meter long radio wave would be three million meters at this scaled) and X-rays and gamma rays would be far too small at this scale.

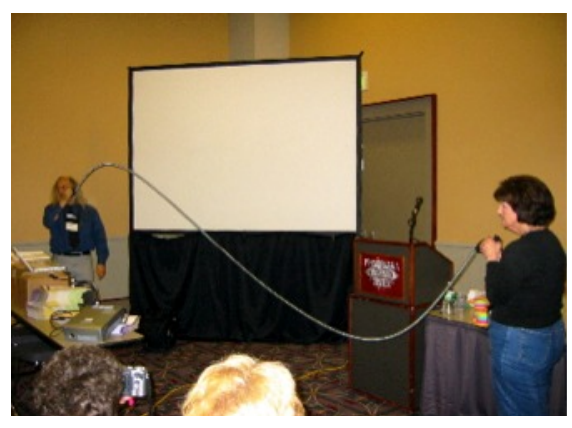

Figure 2: A long spring is used to demonstrate the nature of waves.

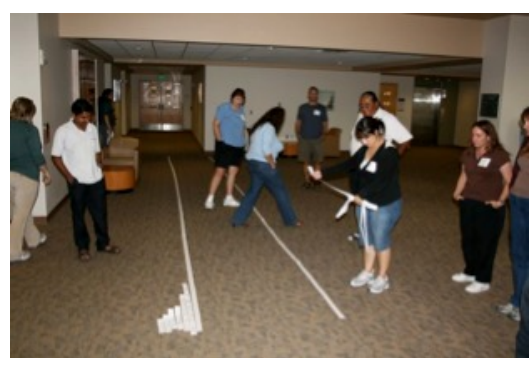

Figure 3: Making a scale model of the EM spectrum.

\subsection{The Kinesthetic Rainbow}

In this activity, students pretend to be different colors of light that make up a rainbow. Each student is given a colored sheet of paper indicating the color of light they represent. Students with blue paper take short quick steps to represent short wavelength light and students with red paper take long slow steps to represent red light while making sure they travel at the same speed. This activity reinforces the inverse relationship between the wavelength and frequency of light. The teacher can reinforce the concept of color filters during this activity by "filtering" out certain wavelengths of light. The teacher holds up a color filter and the students will stop walking when they get to the teacher if their color of light is absorbed by the filter and keep walking if their color of light is transmitted by the filter. Students are reminded of the observations they made in the first activity regarding the filters. Advanced students can be shown filter response curves for different filters before they decide whether their wavelength is transmitted or absorbed. These skills are applied again in the next activity, "Seeing With Alien Eyes".

\subsection{Seeing With Alien Eyes}

This activity is based on a Night Sky Network Activity. Students wear a visor with either a red, blue or green filter. The students are shown a series of different colored felt trees. Working together the green aliens, the red aliens and the blue aliens must use their knowledge of how color filters work to find the green tree. The aliens also must identify other objects such as a yellow Sun and a white cloud. Students must apply their knowledge of the nature of light as well as how filters work as they attempt to determine the true color of various objects.

Astronomers use color filters in their observations. Taking images through different filters let astronomers see different features in objects and can be used to measure the temperatures of objects, learn information about their compositions and estimate the redshifts of distant galaxies. Students can use the color filters provided in the kit to look at various astronomical images and see how they appear through different filters. 


\subsection{Essence of Luminescence}

In this series of activities, students explore various types of luminescence including fluorescence, phosphorescence, chemiluminescence and triboluminescence. An ultraviolet light helps students safely observe fluorescence using invisible ink pens. A Write and See Pad is used in conjunction with a read and a blue LED light to illustrate the different in energies between red and blue light. Glow in the dark stars illustrate the principle of phosphorescence as they keep glowing even after the UV light source has been removed. Glow bracelets are used to illustrate the phenomena of chemiluminescence. Students put the glow bracelets in hot water, cold water and room temperature water and observer the changes in brightness of the glow bracelets. Students explore how changing the temperature of the glow bracelets changes their brightness (which is related to the rate of the chemical reaction). Wint-O-Green Lifesavers let students observe triboluminescence, the light that is given off by the breaking of molecular bonds. Students crush the Wint-OGreen Lifesavers in a dark room and observe the sparks that are produced. Fluorescence is observed in a variety of astronomical objects such as emission nebula.

\section{LIGHT AND COLOR KIT TESTING}

The kit was first used in a teacher workshop at the Astronomical Society of the Pacific (ASP) meeting in Baltimore in August of 2011. 10 teachers signed up for a four-hour workshop using the kit. The kit was well received by the teachers in the workshop. Unfortunately we did not have the resources to conduct follow up with the teachers to see how they used the materials in their classrooms.

In January and February of 2012, all of the activities were used in an afterschool program at the elementary school in Sells, Arizona. The entire sequence of activities was used with a group of primarily fourth and fifth grade students. The entire sequence of activities took six one-hour sessions with the students to complete which is inline with the time estimates in teacher's manual. The materials worked well in this setting. The level of the activities was appropriate for the students and the students were consistently engaged for the entire session. Most of the activities are based on previous well-tested materials.

\section{FUTURE DISTRIBUTION OF THE KITS}

NOAO will produce more kits on an as needed basis for our future teacher workshops. We hope to partner with SPIE in the future to use the kits in short courses at SPIE meetings, focusing on student chapter leaders who will use the kits in outreach efforts at their home institutions.

\section{Acknowledgements}

The National Optical Astronomy Observatory is operated by the Association of Universities for Research in Astronomy (AURA), Inc. under a cooperative agreement with the National Science Foundation.

\section{REFERENCES}

\footnotetext{
1 Pompea, S. M., Walker, C. E., and Sparks, R. T. "Knowledge and Wonder: Engagements with Light and Color in the Hands-On Optics Project," in Exemplary Science in Informal Education Settings: Standards-Based Success Stories, edited by R. Yager and J. Falk, 47-70, NSTA Press (2008)

2 Pompea, S. M., Fienberg, R., Deustua, S., and Isbell, D., "Telescope Kits \& Optics Challenges for the International Year of Astronomy 2009 ", [Education and Public Outreach - A Changing World: Creating Linkages and Expanding Partnerships], Astronomical Society of the Pacific Conference Series 389, eds. C. Garmany, M.G. Gibbs, J.W. Moody, (2008).

3 Pompea, S. M., Dokter, E. F., Walker, C. E., and Sparks, R. T., "Using Misconceptions Research in the Design of Optics Instructional Materials and Teacher Professional Development Programs", Proceedings Education and Training in Optics and Photonics 2007, Ottawa, Canada, (2007)

4 Pompea, S.M. and Gek, T. K., "Optics in the Great Exploration in Math and Science (GEMS) Program: A Summary of Effective Pedagogical Approaches", Proc. SPIE, Vol. 4588 (2002)

5 Pompea, S. M. and Nofziger, M. J., "Resources on Optics in Middle School Education", Proc. SPIE: 1995 International Conference on Education in Optics, Edited by M. J. Soileau, $2525,(1995)$

6 Walker C.E., Pompea S,M, and Sparks R.T. "The Development of an Innovative Ecophotonics/Illumination Engineering Education Program for Grades 6-12” in Proceedings Optics and Photonics (2012)

7 S. M. Pompea, L. W. Fine, and P. Meystre, "Photonics Education for a Green Future: Connecting the Dots of the Arizona STEM Education Experiment, Proceedings SPIE: EcoPhotonics 2011: Sustainable Design, Manufacturing, and Engineering Workforce Education for a Green Future, March 29, 2011, Strasbourg, France
} 
8 C. Walker and S. M. Pompea, "National Education Program for Energy Efficient Illumination Engineering, Proceedings SPIE: Eco-Photonics 2011: Sustainable Design, Manufacturing, and Engineering Workforce Education for a Green Future, March 29, 2011, Strasbourg, France

Proc. of SPIE Vol. $848184810 \mathrm{C}-5$

Downloaded From: https://www.spiedigitallibrary.org/conference-proceedings-of-spie on 26 Apr 2023 Terms of Use: https://www.spiedigitallibrary.org/terms-of-use 